\title{
A 3-D MICROFLUIDIC COMBINATORIAL CELL CULTURE ARRAY
}

\author{
M. C. Liu and Y. C. Tai \\ California Institute of Technology, Pasadena, CA, USA
}

\begin{abstract}
We present the development of a three-dimensional (3-D), on-glass combinatorial cell culture array chip featured with integrated three-input, eight-output combinatorial mixers and cell culture chambers. The device is designed to simultaneously screen for the effects of multiple compounds and concentrations on cultured cells. Experimentally, we first developed a precise way to characterize the combined compound concentration profile at each chamber with a fluorescence method. We then successfully demonstrated the functionality of the cell assay by culturing neuron cells on this device and screening the ability of three chemicals to attenuate cell death caused by cytotoxic hydrogen peroxide. Based on the same technology, the number of inputs and outputs of the combinatorial mixer can be scaled-up to construct labon-chip devices for performing high-throughput cellbased assay and highly parallel and combinatorial chemical or biochemical reactions with reduced labors, reagents, and time.
\end{abstract}

\section{INTRODUCTION}

Microfluidic devices have the potential to become inexpensive platforms for high-throughput cell-based screenings, and recently, many devices with cell culturing and assaying abilities have been developed [1,2]. However, most of the cell culturing devices can only screen for a single compound at once $[3,4]$. A microfluidic device that can screen for the combinatorial effect of multiple compounds on cells is valuable because cells are sustained in complex environments and cell fates are dictated by the integration of numerous extracellular signals. For example, combinatorial effects are evident in cellular processes such as gene regulation [5]. Also, certain combinations of anti-tumor compounds can interact in a synergistic manner on cancer cells [6]. In our previous works, we have demonstrated the method to monolithically fabricate three-dimensional (3-D) microfluidic networks on silicon substrate, and based on this fabrication technology, we have constructed a cell culture device with an integrated combinatorial mixer [7]. In this work, we extended such fabrication method to construct 3-D microfluidic networks on glass substrate and demonstrated a cytotoxic assay using this chip. Although the technology can be extended for more compounds, this work demonstrated a device for three compounds as a feasibility study.

\section{DESIGN}

Figure 1 shows the layout of the $1.7 \mathrm{~cm}$ by $1.7 \mathrm{~cm}$ chip. Compounds $\mathrm{A}, \mathrm{B}$, and $\mathrm{C}$ are flown into eight chambers with different combinations and concentrations depending on the flow resistance of the channels. The overpass structure allows one microfluidic stream to crossover another, and a control channel that receives none of the three inputs is included. The concentration of each compound inside the chamber is determined by the fluidic resistance of the channels. Assuming that the channel width is much larger than the channel height, the resistance can be modeled using the simplified formula for rectangular channel [8]:

$$
R=\frac{12 \mu L}{w h^{3}}
$$

$\mathrm{R}$ is the resistance, $\mu$ is the fluid viscosity, and $\mathrm{L}, \mathrm{w}$, and $\mathrm{h}$ are the length, width and height of the channel, respectively. Chambers receiving multiple compounds are designed to have equal dilution of the input compounds.

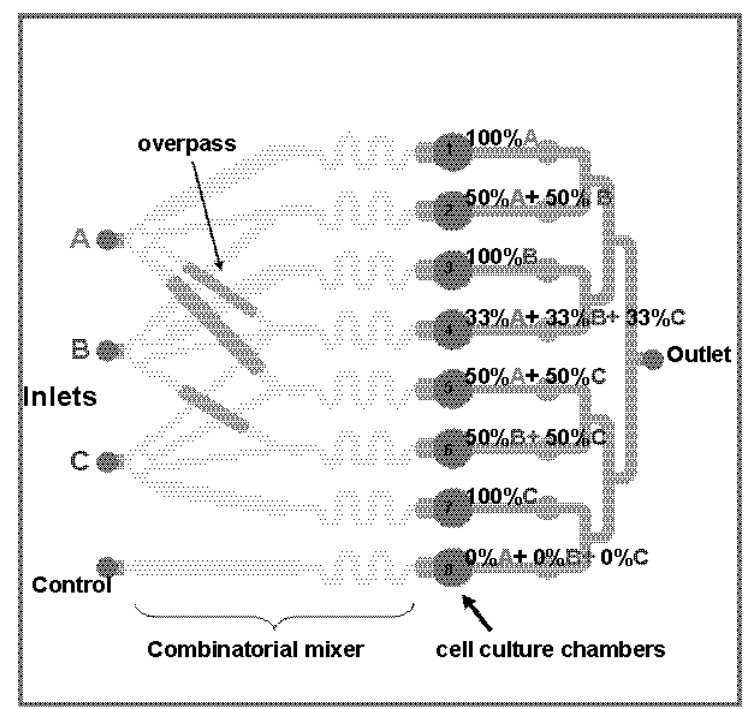

Figure 1. Design Layout of Device

\section{FABRICATION}

The fabrication process for constructing 3-D microfluidic networks is shown in Figure 2. Briefly, a first thin adhesion layer of parylene $\mathrm{C}$ was deposited $(3 \mu \mathrm{m})$ on soda lime glass wafer. The first sacrificial photoresist layer was spin-coated $(14 \mu \mathrm{m})$ and patterned to define the first-level channels. A second layer of parylene C (10 $\mu \mathrm{m})$ was then deposited to cover the sacrificial photoresist, forming the first-level channels. Parylene C was patterned using oxygen plasma so the areas where the overpass structures would be joined were exposed. This parylene patterning also opened the area where the mixer and the culture chamber would be connected. A second sacrificial photoresist was spin-coated $(25 \mu \mathrm{m})$ and patterned to define the overpass structure and the culture chamber. This second sacrificial photoresist covered the areas that were etched open, and the overpasses spanned several of the first-level microfluidic channels. A third layer of parylene $\mathrm{C}(2 \mu \mathrm{m})$ was deposited. The whole chip was planarized with thick SU8 $(90 \mu \mathrm{m})$ and parylene $\mathrm{C}$ was patterned to define the access holes. Finally, the chips were soaked in acetone to dissolve the sacrificial photoresist. 
(a) $1^{\text {st }}$ parylene $C$ deposition on soda lime glass wafer $(3 \mu \mathrm{m})$

$1^{\text {st }}$ sacrificial photoresist coating and patterning $(14 \mu \mathrm{m})$
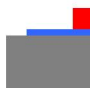

(b) $2^{\text {nd }}$ parylene $C$ deposition and patterning $(10 \mu \mathrm{m})$

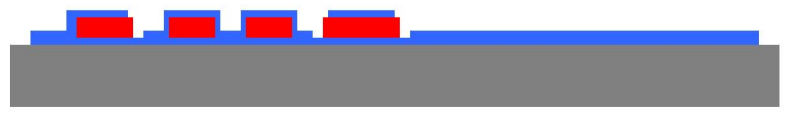

(c) $2^{\text {nd }}$ sacrificial photoresist coating and pattering $(25 \mu \mathrm{m})$

$3^{\text {rd }}$ parylene $C$ deposition $(2 \mu \mathrm{m})$

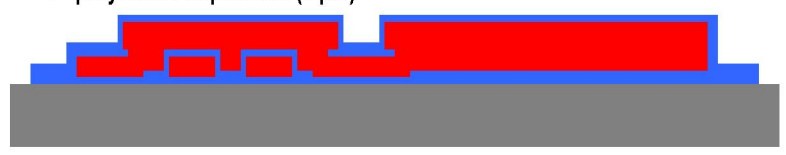

(d) SU8 coating and pattering $(90 \mu \mathrm{m})$

Patterning Parylene $\mathrm{C}$

Sacrificial photoresist stripping in acetone

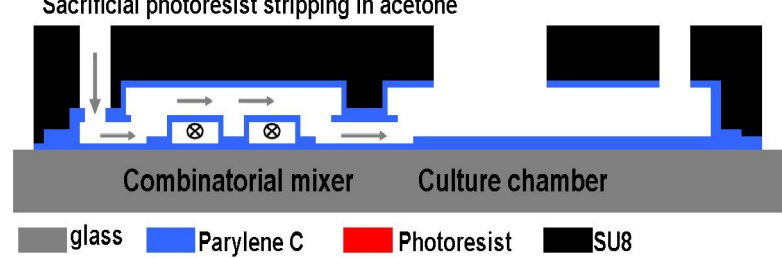

Figure 2. Fabrication process flow. The overpass allows one fluid stream $(\rightarrow)$ to crossover another $(\otimes)$, as shown in $(d)$.

\section{EXPERIMENTAL}

Figure 3 shows the packaging method. A transparent polyester membrane with 0.4 um pores was cut out from Corning ${ }^{\circledR}$ Transwell $^{\circledR}$ cell culture insert, and a rectangular piece of the membrane was placed on top of the chambers. A piece of PDMS (Polydimethylsiloxane) with $100 \mu \mathrm{m}$ height microfluidic networks was fabricated by replicate molding from a SU8 mold. The PDMS was aligned onto the chip and the assembly was clamped together by acrylics. Figure 4 shows the layout of the PDMS piece, which has punched holes that match the locations of the inlet and outlet holes on the parylene microfluidic chip. The PDMS piece also has a cell-seeding inlet and a oneto-eight manifold that distributes cells into eight chambers, which align to the location of the culture chambers of the parylene microfluidic chip. With the membrane, the culture chamber turns into a two-level configuration, and the cell culture level is separated from the fluid delivery level. Syringes were connected with Teflon tubes, which were plugged into the holes of the piece of PDMS.

To characterize the concentration of the three input compounds inside each chamber, we first injected a red fluorescence solution (Sulforhodamine 101) into input A, while injecting DI water into input $\mathrm{B}$, input $\mathrm{C}$ and control, and the fluorescence image of each chamber was captured. Then, the fluorescence intensity was measured using ImageJ. Fluorescence solution was then switched to input $\mathrm{B}$, while injecting DI water into other inlets. The same procedure was repeated for input $\mathrm{C}$. The injection was controlled by syringe pump and was set at $5 \mu \mathrm{L} / \mathrm{min}$ for duration of 20 minutes.

This microfluidic device was used to assay for the ability of 1,5-dihydroxyisoquinoline (ISO), deferoxamine (DFO), and 3-aminobenzoic acid (ABA) to alleviate cytotoxicity of hydrogen peroxide, which is generated during brain ischemia and reperfusion injury and various neuropathological conditions. The device operation for the cell assay experiment is shown in Figure 5. Suspension of rat neuronal cell (B35 rat neuroblastoma cells) was injected, and cells were efficiently collected by and grown on the membrane. Maintaining cells on the membrane and separating them from the fluid delivery channel also mimics the in-vivo tissue environment. Following three hours of incubation after cell seeding, each compound was mixed in media containing $1.5 \mathrm{mM}$ hydrogen peroxide and injected at $5 \mu \mathrm{L} / \mathrm{min}$ for 30 minutes, followed by incubation with normal media for three hours. Live/dead stains (Calcein AM/propidium iodide) were injected, and cytotoxicity was determined by the ratio of dead cells to total cells.

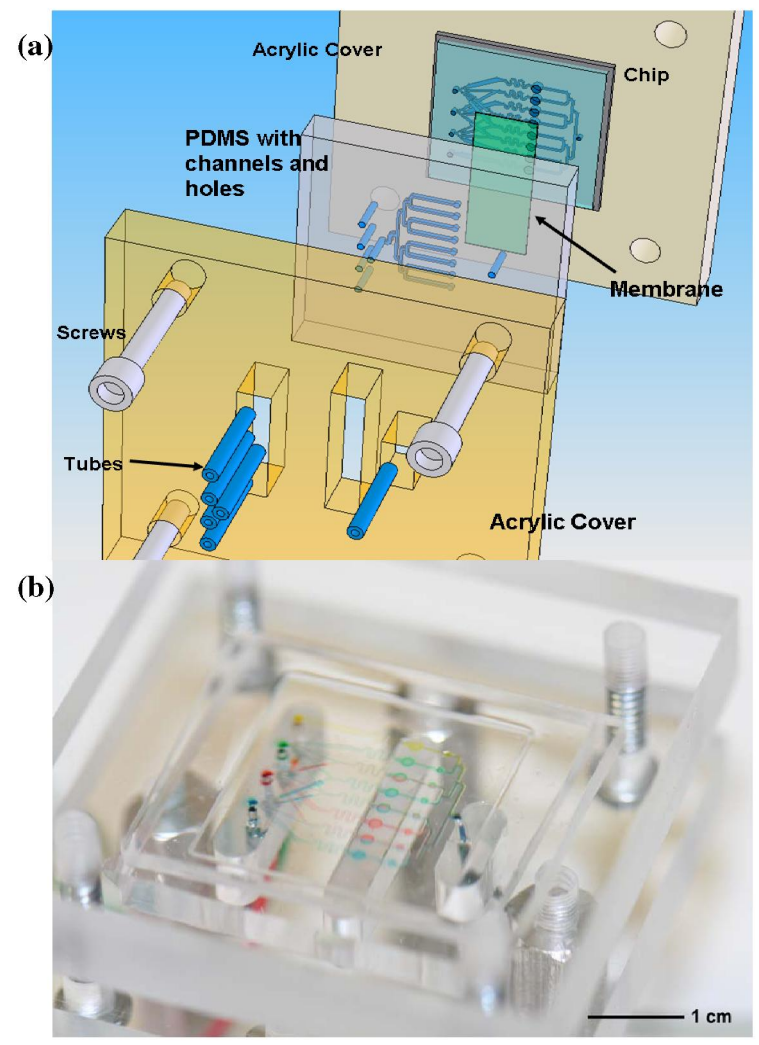

Figure 3. Device packaging. (a) Device packaging schematic. (b) Packaged device with various food coloring solutions injected.

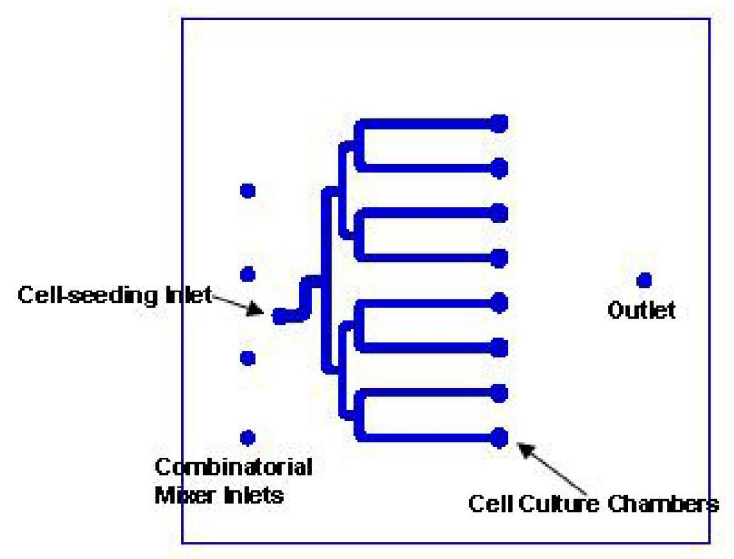

Figure 4. Layout of the PDMS piece. 


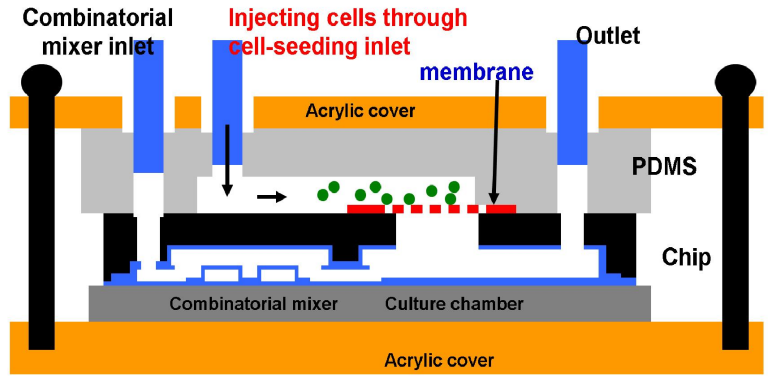

(b) Injecting solutions

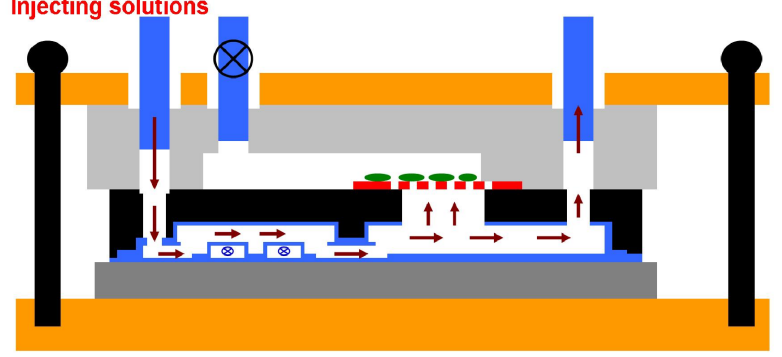

Figure 5. Device operation for cell-based assay. (a) Cell seeding by injecting cell suspension into the cellseeding inlet. The one-to-eight manifold on the PDMS splits the cell suspension and cells flow into the chamber area where they are trapped by the membrane. (b) Injecting various solutions through the combinatorial mixer inlets for cell assay.

\section{RESULTS AND DISCUSSION}

Figure 6 shows the concentration of the three input compounds at each chamber. The combinatorial mixer was able to generate the correct combinations of the compound solutions. The concentration of the compounds can deviate from the design value because of dimensional inaccuracy in processing.

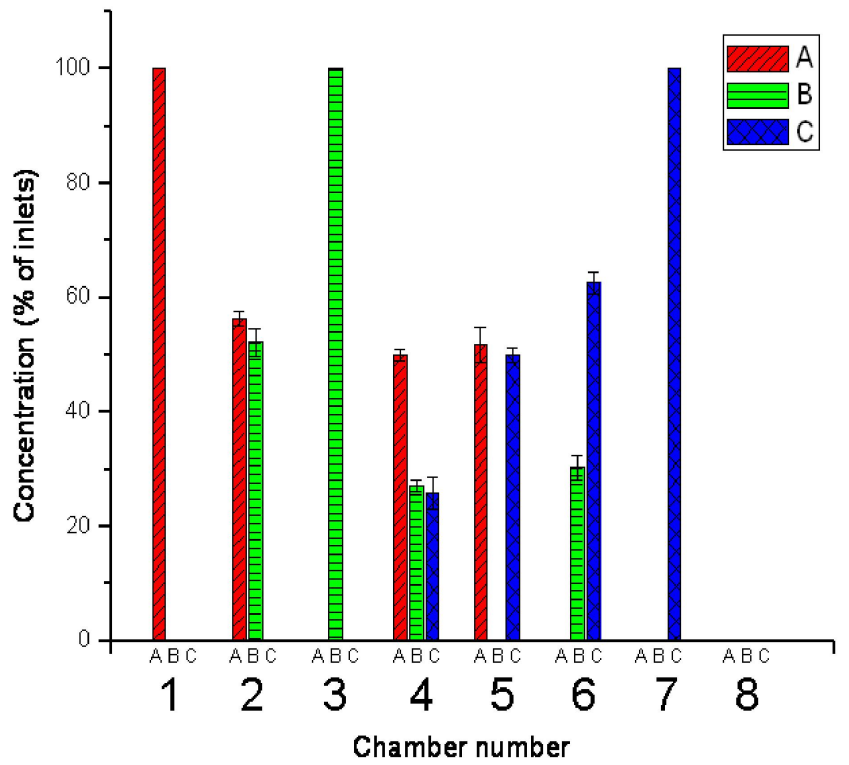

Figure 6. Concentration of the three input compounds at each chamber.

Integrating a porous membrane into the device packaging greatly facilitated the on-chip cell assay experiment. Cell seeding was done without high cell loss as in other microfluidic culture devices that load cells by flowing them through channels. This setup also eliminated the need for coating the channels with cell attachment factors such as collagen or fibronectin. The device was used to assay for effect of three compounds on reducing the cytotoxicity of hydrogen peroxide. Figure 7 shows the result of the cytotoxicity experiment, and Figure 8 shows the arrays of cells after the cells were stained by live/dead stains. 1,5-dihydroxyisoquinoline and deferoxamine showed cytoprotective effect against hydrogen peroxide. 1,5-dihydroxyisoquinoline reduces cell death by inhibiting Poly(ADP-Ribose) polymerase, whose over-production in response to hydrogen peroxide can cause cell death [9]. Deferoxamine reduces cell death by decreasing the conversion of hydrogen peroxide to hydroxyl radicals that damage DNA. 3-aminobenzoic acid, structurally similar to 1,5-dihydroxyisoquinoline, showed minimal effect on hydrogen peroxide cytotoxicity. In general, this device successfully demonstrated that, even with just three inputs, eight distinct cytotoxic experiments were done with different combinatorial compounds using a single chip.

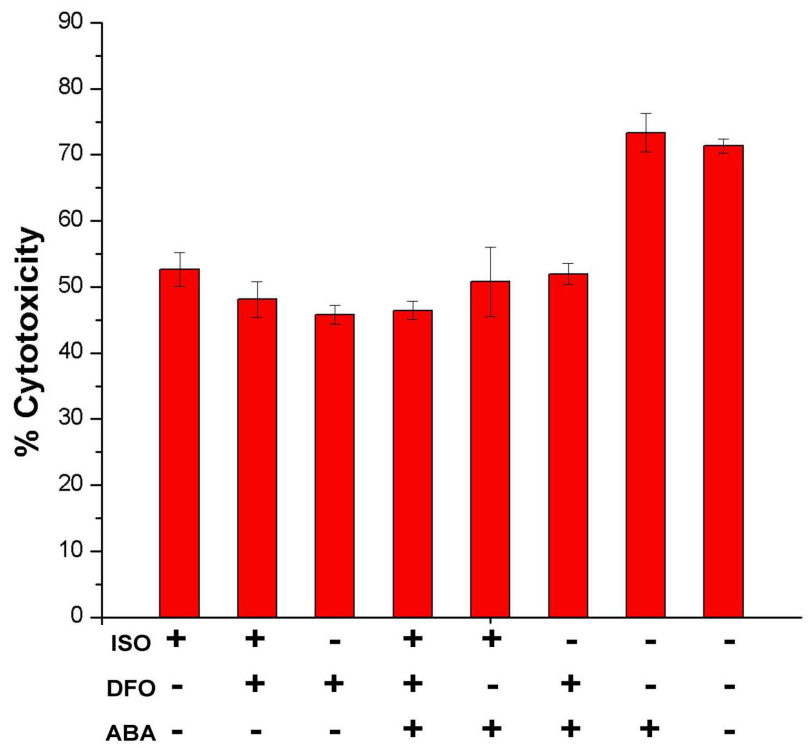

Figure 7. Cytotoxicity as a result of each treatment. The effects of three compounds, 1,5-dihydroxyisoquinoline (ISO), deferoxamine (DFO), and 3-aminobenzoic acid $(A B A)$ on alleviating cell death caused by hydrogen peroxide were tested. Each compound was mixed in media containing $1.5 \mathrm{mM}$ hydrogen peroxide.

\section{CONCLUSION}

In this work, we demonstrated the fabrication of a cell culture chip on glass with integrated combinatorial mixer, and showed a cell-based screening of different combinations of compounds. The combinatorial mixer correctly delivered the distinct combinations of input compounds into the chambers and the concentration of each input compound at the chamber was measured. A porous polyester membrane was incorporated into the culture chamber, and a cytotoxicity experiment was done using this chip. By scaling-up this technology, chip with high-density cell array and integrated high-input combinatorial mixer can be constructed. Such chip will enable inexpensive high-throughput cell-based assay, and significantly benefits research in a spectrum of fields including drug screening and systems biology. 


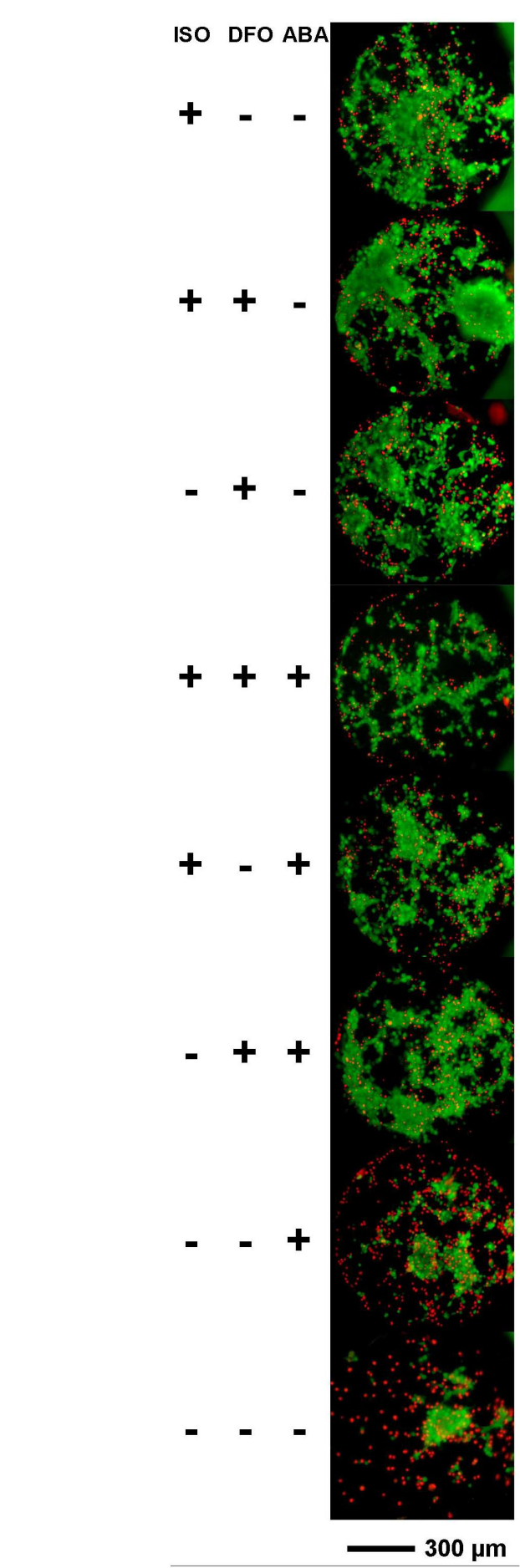

\section{REFERENCES}

[1] L. Kim, Y.C. Toh, J. Voldman and H. Yu, "A practical guide to microfluidic perfusion culture of adherent mammalian cells", Lab Chip, vol. 7, pp. 681-694, 2007. [2] P. J. Lee, P. J. Hung, V. M. Rao and L. P. Lee, "Nanoliter scale microbioreactor array for quantitative cell biology", Biotechnol. Bioeng., vol. 94, pp. 5-14, 2006.

[3] K. R. King, S. Wang, D. Irimia, A. Jayaraman, M. Toner and M. L. Yarmush, "A high-throughput microfluidic real-time gene expression living cell array", Lab Chip, vol. 7, pp. 77-85, 2007.

[4] I. Barbulovic-Nad, H. Yang, P. S. Park and A. R. Wheeler, "Digital microfluidics for cell-based assays", Lab Chip, vol. 8, pp. 519-526, 2008.

[5] S.J. Morrison, N.M. Shah, D.J. Anderson, "Regulatory mechanisms in stem cell biology", Cell, vol. 88, pp. 287298, 1997.

[6] J. A. Menéendez, S. Ropero, M. M. Barbacid, S. Montero, M. Solanas, E. Escrich, H. Cortés-Funes and R. Colomer, "Synergistic interaction between vinorelbine and gamma-linolenic acid in breast cancer cells", Breast Cancer Res. Treat., vol. 72, pp. 203-219, 2002.

[7] M. C. Liu, D. Ho and Y.C. Tai, "Monolithic fabrication of three-dimensional microfluidic networks for constructing cell culture array with an integrated combinatorial mixer", Sens. Actuators B., vol. 129, pp. 826-833, 2008.

[8] G. T. A. Kovacs, Micromachined Transducers Sourcebook, McGraw-Hill Companies, Inc., New York, 1998.

[9] J. Bowes, J. Piper and C. Thiemermann, "Inhibitors of the activity of poly (ADP-ribose) synthetase reduce the cell death caused by hydrogen peroxide in human cardiac myoblasts", Br. J. Pharmacol., vol. 124, pp. 1760-1766, 1998.

Figure 8. Cells in each chamber after live (green)/dead (red) staining. Some early apoptotic cells are stained by both dyes. 